\title{
Levothyroxine with Clomiphene Citrate for Ovulation Induction in Patients with Polycystic Ovary Syndrome and Subclinical Hypothyroidism
}

\author{
Dalia Ibrahim and Ahmed Nofal* \\ Department of Obstetrics and Gynecology, Menoufia University, Egypt
}

Submission: December 14, 2019; Published: January 03, 2020

*Corresponding author: Ahmed Mohammed Nofal, Department of Obstetrics and Gynecology, Menoufia University, Egypt

\begin{abstract}
Introduction: Polycystic ovary syndrome (PCOS), affecting about 5 to $10 \%$ of women [1] Hypothyroid disturbances and elevated TSH levels are common findings in PCOS [2].

Objectives: To study the effect of Eltroxin on ovulatory function in patients with PCO and Subclinical Hypothyroidism (SCH).

Methods: This comparative observational study was conducted on 204 patients with PCO and subclinical hypothyroidism who were subdivided into 2 groups. Control group (1):102 patients who received placebo and Clomiphene Citrate (cc) for 3 successive cycles. Study group (2):102 patients who received Eltroxin and (CC) for 3 successive cycles. Patients were followed up for 3 months for ovulation and pregnancy

Result: There was no significant difference between cases and controls regarding age, parity, BMI. There was no significant difference between both groups regarding ovarian function represented in LH, FSH, TSH, progesterone before treatment. There was significant difference between both groups regarding ovarian function represented in LH, FSH, TSH, progesterone after treatment There was significant difference between both groups regarding ovulation and pregnancy rates.
\end{abstract}

Conclusion: Eltroxin has beneficial effects on ovulation and pregnancy in patients with subclinical hypothyroidism and PCO

Keywords: Eltroxin; Polycystic ovary; Subclinical hypothyroidism

Abbreviatations: SCH: Subclinical Hypothyroidism; CC: Clomiphene Citrate; PCOS: Polycystic Ovary Syndrome; MBS: Metabolic Syndrome; FSH: Follicle Stimulating Hormone; LH: Luteinizing Hormone; DHEA-S: Dehydroepiandrosterone; TSH: Thyroid Stimulating Hormone; USPSTF: United States Preventive Services Task Force

\section{Introduction}

Polycystic ovary syndrome (PCOS), affecting about 5 to $10 \%$ of women, is characterized by hyperandrogenism, menstrual disturbance, obesity, anovulation, infertility, Insulin Resistance, hyperglycemia, weight gain, and metabolic syndrome (MBS) as hypothyroidism [1]. Hypothyroid disturbances and elevated TSH levels are common findings in PCOS. Nanda et, al. [2] 2014 study revealed the prevalence of hypothyroidism in PCOS was $13.26 \%$. Out of that $57.69 \%$ had subclinical hypothyroidism and 42.30 $\%$ had overt hypothyroidism Therefore, women with diagnosed PCOS should be screened for thyroid dysfunction.

Hypothyroidism causes altered follicle stimulating hormone (FSH)/luteinizing hormone (LH) ratio and raised dehydroepiandrosterone (DHEA-S) levels. As excess thyroid stimulating hormone (TSH) causes stimulation of FSH receptor [3]. The National Academy of Clinical Biochemistry suggested serum TSH (0.4- 2.5) $\mathrm{mIU} / \mathrm{L}$ as the normal range, according to United States Preventive Services Task Force (USPSTF) Guidelines defined subclinical hypothyroidism SCH as high serum TSH (2.5-10 mIU/L) with a normal FT4 concentration [4].

Subclinical hypothyroidism is found between 3-8\% of women of reproductive age, has few signs or symptoms of thyroid dysfunction and often remains untreated. Most of the times hypothyroidism is subclinical and diagnosed first time during evaluation of PCOS. In contrast to overt hypothyroidism, where 
most evidence appears to support an association with an increased risk of infertility [4]. The most common endogenous cause of $\mathrm{SCH}$ is chronic autoimmune thyroiditis (Hashimoto's thyroiditis) associated with anti-TPO antibodies, TSH receptor loss of function mutations and presence of heterophile antibodies [5].

Data assessing effect of SCH on fertility are limited due to varied definitions of SCH (different TSH cutoffs) and lack of adequate control groups [4]. In 2015, the American Society for Reproductive Medicine found insufficient evidence to conclude that SCH is associated with infertility [6]. The ATA has issued a weak recommendation that administration of LT4 may prevent progression to overt hypothyroidism once pregnancy is achieved. ASRM recommendations 2015 states that, it is reasonable to screen infertile women for TSH levels, if it is $>4 \mathrm{mIu} / \mathrm{L}$. Patients should be treated with Levothyroxine to maintain TSH level below $2.5 \mathrm{mIu} / \mathrm{L}$ (Grade B), If TSH is between $2.5-4 \mathrm{mIu} / \mathrm{L}$ management options either observation or giving Levothyroxine (Grade C).

CC resistance represents up to $20-40 \%$ of infertile women with PCOS. in one study, a total of 145 PCOS patients were received CC stimulating test. One hundred and twelve patients had ovulation, with an ovulation rate of $77.2 \%, 35$ patients $(22.8 \%)$ CC resistant. Logistic regression revealed a significant association between SCH and CC resistance. The coexistence of PCOS and SCH has contributed to a clearly higher prevalence of CC resistance (30.4\%) than that in the Euthyroid (EU) group (14.5\%), this result has demonstrated that $\mathrm{SCH}$ would impact the response of ovulation induction with CC in PCOS patients [7].

\section{Patients and Methods}

This was a prospective observational study that was conducted at infertility unit in menoufia university hospitals upon patients suffering from infertility, PCOS and subclinical hypothyroidism from June 2018 till may 2019. 220 patients enrolled into this study ,18 cases dropped out of the study due to failure of follow up of the patients, the 204 patients who completed the study were randomized by random randomization into 2 groups Group (I) in this group102 patients received 50u Eltroxin till TSH was normalized then they received combined Eltroxin and 100mg Clomid for 3 successive cycles Group (II) 102 patients received sugary tablet and $100 \mathrm{~m}$ Clomid for 3 successive cycles.

Ethical approval of the study protocol was obtained from Menoufia faculty of Medicine (Ethical clearance letter number 319G/2018) in accordance with the revised Helsinki declaration. All participants signed the informed consent form before commencing the study.

\section{Sample Size}

The STATCALC feature of Epi Info ${ }^{\mathrm{TM}}$-Version 6 software was used for calculating the sample size guided by:

Power of the test $=80 \%$, Confidence level $=95 \%$, Accepted margin of error $=5 \%$ Risk percent ratio $=2.5 \%$, Expected frequency of condition $=10 \%$, Total sample accepted according to inclusion criteria $=100$. Type of the study: cross sectional observational study. Calculation of the sample size was based on the results of the previous observational studies.

\section{Inclusion Criteria}

Infertile females with PCOS and SCH. PCOS were diagnosed according to the criteria of the Rotterdam, European Society of Human Reproduction and Embryology-American Society for Reproductive Medicine-sponsored PCOS consensus workshop group (59T759T). Patients with two out of the following three features will be considered as PCOS: oligoovulation and/or anovulation, clinical and/ or biochemical signs of hyperandrogenism, and polycystic ovaries on ultrasound examination (the presence of 12 or more follicles measuring $2-9 \mathrm{~mm}$ in diameter). Oligo/anovulation was defined as the presence of oligomenorrhea (menstrual cycles of $>35$ days) or amenorrhea (lack of menstrual period for 6 months or more) [7]. Sub clinical hypothyroidism was defined as $\mathrm{TSH}>2.5 \mathrm{mIU} / \mathrm{L}$ and normal thyroid hormones [4].

\section{Exclusion Criteria}

Patients who had known history of diabetes mellitus, hyper prolactinemia, congenital adrenal hyperplasia, hypothyroidism or hyperthyroidism, Cushing's disease, renal, hepatic, or cardiac dysfunction, a history of ovarian or adrenal neoplasm, and those using any medication (e.g. oral contraceptives, insulin-sensitizing drugs, statins, radioactive Iodine, Levothyroxine, corticosteroids and GnRH agonists and antagonists) within 6 months of the enrollment to the study were excluded.

\section{Methods}

Complete systemic clinical examination and anthropometric assessment including height, weight, body mass index (BMI), waist and hip circumferences was done for every patient by a single person and with the same instruments in the clinic so that the inter observer errors were limited. $5 \mathrm{~mL}$ of venous blood were obtained and used for estimation of fasting plasma glucose, lipid profile, and hormonal analysis. Blood sample were taken on day 2 or 3 of the menstrual cycle (21for progesterone).

Hormonal analysis included serum free triiodothyronine (T3), free tetraiodothyronine (T4), TSH, anti-thyroperoxidase antibody (anti-TPO ab), LH, FSH, prolactin, insulin, free testosterone, progesterone (assessed at day 21 postmenstrual), estradiol, and SHBG-S using Cobas e-411 analyzer (Roche Diagnostics Ltd., Mannheim, Germany) by electrochemiluminescence immunoassay (ECLIA) method as per manufacturer's protocol (intra-assay and interassay $\mathrm{CV}<5.0 \%$ ). Lipid profile and plasma glucose were estimated on Cobas c-311 clinical chemistry analyzer (Roche Diagnostics Ltd., Mannheim, Germany) by standard methods as per manufacturer's protocol. Group (I) in this group patients received 50u Eltroxin till TSH was normalized then they received 
combined Eltroxin and 100mg Clomid for 3 successive cycles Group (II) patients received sugary tablet and $100 \mathrm{~m}$ clomid for 3 successive cycles the patients were followed up for detection of ovulation (folliculometry) and clinical pregnancy by transvaginal ultrasound. Hormonal profile was repeated after treatment for both groups.

\section{Statistical Analysis}

Data were tabulated and analyzed by SPSS version 20 on IBM compatible computer.

\section{Result}

There was no significant difference between cases and controls regarding age, parity, BMI (table 1) There was significant difference between both groups regarding ovarian function represented in LH, FSH, TSH, progesterone before and after treatment (table 2).There was significant difference between both groups regarding ovulation and pregnancy rate as in (table 3). Comparison between both groups before treatment (table 4).

Table 1: Demographic criteria.

\begin{tabular}{|c|c|c|c|c|}
\hline & Group (1) & Group (2) & T test & $P$ value \\
\hline Age & $30.44+4.96$ & $28.96+4.95$ & 2.49 & 0.013 \\
\hline BMI & $35.9+40.35$ & $31.03+28.7$ & 0.9 & 0.32 \\
\hline \multirow[t]{2}{*}{ Free and gallaway score } & $16.7+8.6$ & $15.6+8.2$ & 0.86 & 0.37 \\
\hline & & & Chi square & \\
\hline \multicolumn{5}{|l|}{ Hypertension } \\
\hline Yes & $43(42.6 \%)$ & $49(48.5 \%)$ & 0.719 & 0.48 \\
\hline No & $58(57.4 \%)$ & $52(51.5 \%)$ & & \\
\hline \multicolumn{5}{|l|}{ Hirsutism } \\
\hline Yes & $62(61 \%)$ & $65(64 \%)$ & 0.191 & 0.77 \\
\hline no & $38(39 \%)$ & $36(36 \%)$ & & \\
\hline
\end{tabular}

Table 2: laboratory characteristics of control group.

\begin{tabular}{|c|c|c|c|c|c|c|}
\hline & \multicolumn{2}{|c|}{ Mean } & \multicolumn{2}{|c|}{ Standard Deviation } & \multirow{2}{*}{ T test } & \multirow{2}{*}{$P$ value } \\
\hline & Before & After & Before & After & & \\
\hline FSH-FSH after & 5.59 & 5.599 & 1.2 & 1.3 & 1 & 0.32 \\
\hline HOMA-IR - HOMA-IR after & 4.56 & 4.51 & 0.89 & 0.86 & 1.26 & 0.207 \\
\hline Insulin - insulin after & 13.6 & 13.5 & 2.08 & 2.01 & 1 & 0.32 \\
\hline Estradiol (pg/mL) - estradiol after & 65.8 & 65.5 & 11.7 & 12.2 & 0.381 & 0.704 \\
\hline TSH -TSH after & 3.7 & 3.4 & 4.2 & 2.9 & 0.568 & 0.571 \\
\hline Progesterone $(\mathrm{ng} / \mathrm{mL})$-progest after & 2.08 & 3.16 & 0.31 & 2.7 & 4.02 & 0.0005 \\
\hline
\end{tabular}

Table 3: laboratory characteristics of Study group.

\begin{tabular}{|c|c|c|c|c|c|c|}
\hline \multirow{2}{*}{} & \multicolumn{2}{|c|}{ Mean } & \multicolumn{2}{c|}{ Standard Deviation } & \multirow{2}{*}{ T test } \\
\cline { 2 - 6 } & Before & After & Before & After & & \\
\hline LH - LH af & 11 & 8.5 & 2.5 & 1.3 & 11.04 & 0.0005 \\
\hline FSH - FSH af & 5.54 & 5.48 & 1.28 & 1.28 & 2.07 & 0.04 \\
\hline HOMA-IR - HOMA-IR af & 4.523 & 2.23 & 0.86 & 0.71 & 19.48 & 0.0005 \\
\hline Insulin - insulin af & 13.52 & 9.99 & 2.02 & 2.22 & 11.34 & 0.0005 \\
\hline Estradiol (pg/mL) - estradiol af & 65.07 & 65.07 & 11.56 & 46.72 & 12.24 & 0.0005 \\
\hline TSH - TSH aft & 3.7 & 1.49 & 4.25 & 0.44 & 5.34 & 0.0005 \\
\hline Progesterone (ng/mL) - prog aft & 6.6 & 20.8 & 0.3 & 4.5 & 10 & 0.0005 \\
\hline
\end{tabular}


Table 4: Comparison between both groups regarding.

\begin{tabular}{|c|c|c|c|c|}
\hline & Group1 & Group2 & Chi Square & P Value \\
\hline $\begin{array}{c}\text { Ovulation } \\
\text { Yes } \\
\text { No }\end{array}$ & $61(60.4 \%)$ & $21(20.8 \%)$ & 32.8 & 0.0005 \\
\hline $\begin{array}{c}\text { Pregnancy } \\
\text { Yes }\end{array}$ & $\begin{array}{c}21(20.8) \\
\text { No }\end{array}$ & $\begin{array}{c}7(6.9 \%) \\
90(79.2 \%)\end{array}$ & 8.12 & 0.004 \\
\hline
\end{tabular}

\section{Discussion}

Hypothyroidism can interfere with gonadotropin secretion by elevating serum prolactin levels [8]. Clinical manifestations include menstrual irregularities and infertility due to anovulation and/or luteal phase defect, an association has been reported between PCOS and hypothyroidism. hypothyroidism may be subclinical and diagnosed first time during evaluation of PCOS [7]. Probable mechanism of subclinical hypothyroidism in PCOS may be obesity and high BMI. proinflammatory condition and IR, may lead to decreased deiodinase- 2 activity and relative low T3 and high TSH levels [9-10]. Alternate mechanism is increased leptin levels stimulating hypothalamus to increase TRH secretion [9]. one or both pathways may be the cause $[10,11]$.

Data assessing the effect of SCH on fertility are limited due to varied definitions of SCH (different TSH cutoffs) and limited control groups [4]. In one study, a total of 145 PCOS patients were received $\mathrm{CC}$, with an ovulation rate of $77.2 \%$, and $(22.8 \%)$ were CC resistant. Logistic regression revealed a significant association between SCH and CC resistance. The coexistence of PCOS and SCH has contributed to a clearly higher prevalence of CC resistance (30.4\%) than that in the EU group (14.5\%), with the OR of 2.645 (95\%CI: 1.122-6.239). This result has demonstrated that SCH would hamper ovulation induction with CC in PCOS patients [7]. There are two possible explanations for this phenomenon. First, SCH is common in patients with Hashimoto' thyroiditis (HT) with high anti TPO which dramatically increases risk of CC resistant. Second, even a short-term of lifestyle modification had reproductive benefits in overweight and obese patients with PCOS who were resistant to CC [7].

A retrospective study of 394 women found a significantly higher incidence of SCH affecting $13.9 \%$ of infertile women compared with controls (3.9\%) [12]. This current study included 204 patients divided into 2 groups: Group (I) in this group patients received 50u Eltroxin till TSH was normalized then they received combined Eltroxin and 100mg Clomid for 3 successive cycles Group (II) patients received sugary tablet and $100 \mathrm{~m}$ Clomid for 3 successive cycles.

The patients were followed up for detection of ovulation (folliculometry) and clinical pregnancy by transvaginal ultrasound.
Hormonal profile was repeated after treatment for both groups. There was no significant difference between cases and controls regarding age, parity, BMI while, there was no significant difference between both groups regarding ovarian function represented in LH, FSH, TSH, progesterone before treatment there was significant difference regarding hormonal levels after and before treatment in both groups. There was significant difference between both groups regarding ovulation and pregnancy rate after treatment. These results suggest that Eltroxin has beneficial effects on ovulation and pregnancy in patients with subclinical hypothyroidism and PCO.

In accordance to the results of this study there was previous studies confirm these results as In Jatzko study about The impact of thyroid function on intrauterine insemination outcome, there was no significant changes as regard demographic criteria as age BMI, hypertension, and hirsutism in both groups .there were highly significant changes as regarding LH,FSH,TSH,HOMA IR and insulin before and after administration of thyroxin in the study group however there was insignificant changes in control group . There were highly significant changes as regarding level of progesterone before and after administration of thyroxine in the study group but no significant difference in control group. there were highly significant changes as regarding the rate of ovulation and pregnancy in the study group but insignificant changes in control group [13].

There are few studies assessing whether LT4 therapy improves outcomes for infertile women with SCH not undergoing assistive reproductive techniques [4]. A retrospective study examining LT4 therapy on 69 infertile women reported 84\% pregnancy rate with treatment [14]. In the study by Bohnet et al. [15]. treatment with L-thyroxine $50 \mathrm{ì} \mathrm{g} /$ day had normalized mid progesterone secretion and two among the eleven SCH treated women became pregnant.

Raber et al. [16] Investigated a group of 283 women with infertility. All patients underwent a TRH stimulation test $\mathrm{SCH}$ was defined as a serum TSH $>15 \mathrm{mU} / \mathrm{L}$ ). Women with a diagnosis of SCH were treated with thyroxine and followed prospectively over a 5-year period. Among these women $34 \%$ had SCH. women who achieved a basal serum TSH $<2.5 \mathrm{mU} / \mathrm{L}$ or a TRH stimulated $\mathrm{TSH}<20 \mathrm{mU} / \mathrm{L}$ became pregnant more frequently. Randomized controlled trials showed that LT4 therapy improved pregnancy in women with SCH undergoing artificial reproductive techniques [11].

A randomized non-placebo controlled trial reported that after treatment with L-thyroxine, 64 infertile women with SCH (TSH $>4.5 \mathrm{mIU} / \mathrm{L}$ ) undergoing IVF/intracytoplasmic sperm injection. The 32 women randomized to $50 \mu$ g of LT4 with a goal to reach and maintain TSH of $<2.5 \mathrm{mIU} / \mathrm{L}$ had a lower miscarriage rate $(0 \%$ vs $33 \%, \mathrm{P}=0.02)$ and higher live birth rate $(53 \%$ vs $25 \%, \mathrm{P}=0.04)$ 
and a significantly higher embryo implantation rate (RR:1.8, 1.003.25; $\mathrm{P} 1 / 4$ 0.05) compared with the control group [17]. women with SCH (TSH $>4.0 \mathrm{mIU} / \mathrm{L}$ ) undergoing in vitro fertilization (IVF)/ intracytoplasmic sperm injection were randomized to $50 \mu \mathrm{g}$ of LT4 therapy with a goal to normalize TSH before IVF or placebo (35 women in each group). The LT4 arm had a lower miscarriage rate ( $9 \%$ vs $13 \%$ for control, $\mathrm{P}=0.03)$ and a higher live birth rate $(26 \%$ vs $3 \%$ for control, $\mathrm{P}=0.02$ ) [18].

On contrary. A prospective study of 538 women showed that the mean TSH level was statistically higher in infertile women (1.3mIU/L) compared with controls $(1.1 \mathrm{mIU} / \mathrm{L})$, but this difference did not appear clinically significant. Additionally, the study found no difference in the prevalence of TSH $>4.2 \mathrm{mIU} / \mathrm{L}$ in the infertile group vs the control group [3]. Similarly, a cross-sectional study analyzed the sera of 704 women undergoing infertility treatment and found only 16 with a raised TSH level (2.3\%), which is similar to general population rates [19]. Moreover, a prospective cohort study found that among healthy fecund women with a history of pregnancy loss, a TSH level $\geq 2.5 \mathrm{mIU} / \mathrm{L}$ was not associated with fecundity, pregnancy loss, or live birth [10].

A large prospective study of 270 patients with SCH undergoing IVF who received LT4 therapy showed. that there may be no benefit in strictly controlling TSH levels $(<2.5 \mathrm{mIU} / \mathrm{L})$ for those women with $\mathrm{SCH}$ receiving LT4 therapy who are undergoing assistive reproductive techniques.as similar miscarriage and live birth rate between those who achieved a target TSH level of 0.2 to $2.5 \mathrm{mIU} / \mathrm{L}$ compared with a TSH level of 2.5 to $4.2 \mathrm{mIU} / \mathrm{L}$ [20].

\section{Limitation}

Inability to extend the period of follow up to record the clinical outcome of the pregnant patients as well as the low number of patients, constitute unintended limitations of the current study.

\section{Conclusion}

Eltroxin has beneficial effects on ovulation and pregnancy in patients with subclinical hypothyroidism and PCO.

\section{Acknowledgment}

All authors have contributed significantly, and agree with the content of the manuscript.

\section{References}

1. Yue-Hong Lu, Zhou-Lan Xia, Ying-Ying Ma, Hui-Juan Chen, Li-Ping Yan, et al. (2016) Subclinical hypothyroidism is associated with metabolic syndrome and clomiphene citrate resistance in women with polycystic ovary syndrome. Gynecol Endocrinol 32(10): 852-855.

2. Sudhanshu S, Subhalaxmi D, Ashok B, Bharati M (2014) Thyroid Profile in Polycystic Ovarian Syndrome. Journal of Evolution of Medical and Dental Sciences 37(3): 9594-9600.

3. Poppe K, Velkeniers B, Glinoer D (2007) Thyroid disease and female reproduction. Clin Endocrinol 66(3): 309-321.
4. Spyridoula M, Naykky M, Singh O, George M, Derek T, et al. (2018) Subclinical Hypothyroidism in Women Planning Conception and During Pregnancy: Who Should Be Treated and How? J Endocr Soc 2(6): 533-546.

5. Papi G, Uberti ED, Betterle C, Carani C, Pearce EN, et al. (2007) Subclinical hypothyroidism. Curr Opin Endocrinol Diabetes Obes 14(3): 197-208.

6. American society for reproductive medicine (2015) subclinical hypothyroidism in the infertile female population: a guideline. fertility sterility 104(3): 545-553

7. Cristina L, Vanessa R, Heraldo M (2013) Subclinical hypothyroidism in young women with polycystic ovary syndrome: an analysis of clinical, hormonal, and metabolic parameters. Fertile Steril 99(2): 588-592.

8. Vissenberg R, Boogaard E, Wely M, Post J, Fliers E, et al. (2012) Treatment of thyroid disorders before conception and in early pregnancy: a systematic review. Human Reprod Update 18(4): 360373.

9. Poppe K, Glinoer D, Steirteghem VA, Tournaye H, Devroey P, et al. (2002) Thyroid dysfunction and autoimmunity in infertile women. Thyroid 12(11): 997-1001.

10. Plowden C, Schisterman F, Sjaarda A, Zarek M, Perkins J, et al. (2016) Subclinical hypothyroidism and thyroid autoimmunity are not associated with fecundity, pregnancy loss, or live birth. J Clin Endocrinal Metab 101(6): 2358-2365.

11. Qun Y, Jin-B (2016) Subclinical Hypothyroidism in PCOS: Impact on Presentation, Insulin Resistance, and Cardiovascular Risk. BioMed Research International, pp. 7-14.

12. Abalovich M, Mitelberg L, Allami C, Gutierrez S, Alcaraz G, et al. (2007) Subclinical hypothyroidism and thyroid autoimmunity in women with infertility. Gynecol Endocrinol 23(5): 279-283.

13. Birgit J, Elisabeth V, Alexandra P, Regina P, Klaus M, et al. (2014) The impact of thyroid function on intrauterine insemination outcome - a retrospective analysis. Reprod Biol Endocrinol 12: 28.

14. Yoshioka W, Amino N, Ide A, Kang S, Kudo T, et al. (2015) Thyroxine treatment may be useful for subclinical hypothyroidism in patients with female infertility. Endocr J 62(1): 87-92.

15. Bohnet G, Fiedler K, Leidenberger A (1981) Subclinical hypothyroidism and infertility. Lancet 2(8258): 1278.

16. Raber W, Nowotny P, Vytiska-Binstorfer E, Vierhapper H (2003) Thyroxine treatment modified in infertile women according to thyroxine-releasing hormone testing: 5- year follow-up of 283women referred after exclusion of absolute causes of infertility. Human Reproduction 18(4): 707-714.

17. Kim H, Ahn W, Kang P, Kim H, Chae D, et al. (2011) Effect of levothyroxine treatment on in vitro fertilization and pregnancy outcome in infertile women with subclinical hypothyroidism undergoing in vitro fertilization/intracytoplasmic sperm injection. Fertil Steril 95(5): 1650-1654.

18. Abdel Rahman A, Aly H, Abbassy A (2010) Improved in vitro fertilization outcomes after treatment of subclinical hypothyroidism in infertile women. Endocr Pract 16(5): 792-797.

19. Llincoln S, Ke R, kutteh W (1999) Screening for hypothyroidism in infertile women. J reprod med 44(5): 455-557.

20. Cai Y, Zhong L, Guan J, Guo R, Niu B, et al. (2017) Outcome of in vitro fertilization in women with subclinical hypothyroidism. Reprod Biol Endocrinol 15(1): 39. 


\section{Your next submission with Juniper Publishers} will reach you the below assets

- Quality Editorial service

- Swift Peer Review

- Reprints availability

- E-prints Service

- Manuscript Podcast for convenient understanding

- Global attainment for your research

- Manuscript accessibility in different formats

( Pdf, E-pub, Full Tsext, Audio)

- Unceasing customer service

Track the below URL for one-step submission https://juniperpublishers.com/online-submission.php 